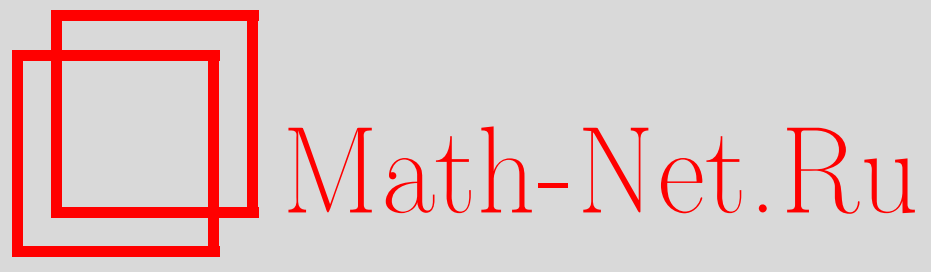

Н. Ф. Зак, Квазитривиальность форм многообразий Сегре, УМH, 2007, том 62, выпуск 5, 153-154

DOI: https://doi.org/10.4213/rm7484

Использование Общероссийского математического портала Math-Net.Ru подразумевает, что вы прочитали и согласны с пользовательским соглашением http://www . mathnet.ru/rus/agreement

Параметры загрузки:

IP: 35.173 .219 .12

26 апреля 2023 г., 13:23:32

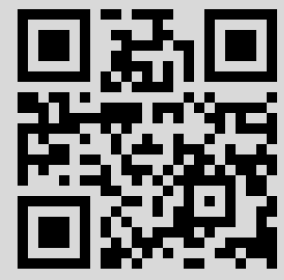




\section{Квазитривиальность форм многообразий Сегре}

\section{Н. Ф. Зак}

Пусть $X$ - многообразие, определенное над полем $\mathbb{k}$ характеристики нуль. Соответствующее многообразие $X \times_{\mathbb{k}} \overline{\mathbb{k}}$ над алгебраическим замыканием $\overline{\mathbb{k}}$ поля $\mathbb{k}$ мы будем обозначать через $\bar{X}$, говоря, что $X$ является формой $\bar{X}$ и всех изоморфных ему над $\overline{\mathbb{k}}$ многообразий. Пусть $G=\operatorname{Gal}(\overline{\mathbb{k}} / \mathbb{k})$ - группа Галуа. В [1]) доказывается

УтвеРЖДЕниЕ 1. Пусть на многообразии $X$ есть $\mathbb{k}-$ точка. Тогда имеет место изоморфизм групп $\operatorname{Pic}(X) \simeq \operatorname{Pic}^{G}(\bar{X})$ и всякая Галуа-инвариантная линейная система порождается дивизорами, определенными над $\mathbb{k}$. Кроме того, Галуа-инвариантная часть очень обильной линейной системь задает вложение над $\mathbb{k}$.

Хорошо известно (см. [2]), что всякая форма $X$ проективного пространства $\mathbb{P}^{n}$ квазитривиальна, более того, теорема Севери-Брауэра утверждает, что из наличия $\mathbb{k}$-точки на $X$ следует изоморфизм $X \simeq_{\mathbb{k}} \mathbb{P}^{n}$. Аналогичный результат неверен для произведений проективных пространств. Мы докажем следующее утверждение.

Теорема 2. Всякая форма $X$ многообразия $S=\mathbb{P}^{a_{1}} \times \cdots \times \mathbb{P}^{a_{n}}$ квазитривиальна.

Рациональность $S$ можно (хотя, конечно, это далеко не простейший способ) доказать с помощью следующего предложения.

Предложение 3. Рассмотрим стандартное вложение $S$ по Сегре. Выберем 8 каждом $i$-м сомножителе гиперплоскость $H_{i}$ и рассмотрим подмногообразия $\mathscr{P}_{i j}=$ $\mathbb{P}^{a_{1}} \times \cdots \times \mathbb{P}^{a_{i-1}} \times H_{i} \times \mathbb{P}^{a_{i+1}} \times \cdots \times \mathbb{P}^{a_{j-1}} \times H_{j} \times \mathbb{P}^{a_{j+1}} \times \cdots \times \mathbb{P}^{a_{n}}, \quad i<j$. Проекция $\pi_{L}: S \rightarrow \mathbb{P}^{\sum a_{i}}$ из проективной оболочки $L$ объединения $\mathscr{P}=\bigcup \mathscr{P}_{i j}$ бирачиональна. $B$ частности, если $S$ и L определены над $\mathbb{k}$, то проекция $\pi_{L}$ бирациональна над $\mathbb{k}$.

ДокАзАтельство. Конечность проекции $\pi_{L}: S \rightarrow \mathbb{P}^{\sum a_{i}}$ проверяется прямым подсчетом размерности $L$. Заметим, что через любые две точки $p, q \in S$ проходит подмногообразие $S_{1}=T_{1} \times \cdots \times T_{n} \subset S$, где $T_{i}=\mathbb{P}^{1} \subset \mathbb{P}^{a_{i}}$, поэтому, ввиду конечности общего слоя $\pi_{L}$, достаточно доказать бирациональность проекции $\pi_{L}: S_{1} \rightarrow \mathbb{P}^{n}$ для общей точки $p$ и произвольной точки $q$. Заметим, что в этом случае $\mathscr{P} \cap S_{1}=\bigcup P_{i j}$ и, более того, $L \cap S_{1}=\bigcup P_{i j}$, где

$$
P_{i j}=T_{1} \times \cdots \times T_{i-1} \times p_{i} \times T_{i+1} \times \cdots \times T_{j-1} \times p_{j} \times T_{j+1} \times \cdots \times T_{n}, \quad i<j,
$$

а $p_{i}=H_{i} \cap T_{i}$. Следовательно, нам достаточно проверить бирациональность проекции $\pi_{L_{1}}: S_{1} \rightarrow \mathbb{P}^{n}$, где $L_{1}-$ линейная оболочка объединения $P=\bigcup P_{i j}$. Рассмотрим общую точку $q=q_{1} \times \cdots \times q_{n} \in S_{1}$. Пусть $Q_{i}=T_{1} \times \cdots \times T_{i-1} \times q_{i} \times T_{i+1} \times \cdots \times T_{n}$, a $P_{j}=T_{1} \times \cdots \times T_{j-1} \times p_{j} \times T_{j+1} \times \cdots \times T_{n}$. Рассмотрим $n$ линейно независимых гиперплоских сечений $\mathscr{H}_{i} \subset S_{1}$, содержащих $L_{1}$ и $q: \mathscr{H}_{i}=Q_{i} \cup P^{i}$, где $P^{i}=\bigcup_{j \neq i} P_{j}$. Пусть $\mathscr{H}=\bigcap \mathscr{H}_{i}$. Тогда бирациональность проекции $\pi_{L_{1}}$ эквивалентна тому, что $\mathscr{H} \cap S_{1}=\{q\} \cup P$. Рассмотрим точку $r=r_{1} \times \cdots \times r_{n} \in \mathscr{H} \cap S_{1}$. Поскольку $r \in Q_{i} \cup P^{i}$, то либо $r_{i}=q_{i}$, либо $r_{k}=p_{k}$ для некоторого $k$. Если для всех $i$ справедлива первая альтернатива, то $r=q$. Если же $r_{k}=p_{k} \neq q_{k}$, то, поскольку $r \in Q_{k} \cup P^{k}$, для некоторого $l \neq k$ выполнено $r_{l}=p_{l}$, а значит, $r \in P_{k l} \subset P$, что доказывает бирациональность $\pi_{L_{1}}$.

ДокАЗАТЕЛЬСтво теоремы 2. Поскольку группа Галуа не может переставлять стягивания на многообразия разных размерностей, $\mathbb{k}$-форма $X$ многообразия $S$ изоморфна произведению $\mathbb{k}$-форм многообразий $S_{i}=\mathbb{P}^{a_{i}} \times \cdots \times \mathbb{P}^{a_{i}}$ и нам достаточно доказать квазитривиальность этих сомножителей. Рассмотрим многообразие $S=$ $\mathbb{P}^{n} \times \cdots \times \mathbb{P}^{n}$. Линейная система, вкладывающая $S$ по Сегре, пропорциональна каноническому классу $K_{S}$, а значит, Галуа-инвариантна. Поэтому, согласно утверждению 1 ,

Работа выполнена при поддержке РФФИ (грант № 04-01-00613). 
можно считать, что $\bar{X}$ вложено по Сегре. Мы построим Галуа-инвариантное объединение $\bigcup H_{i}$, тогда $\bigcup \mathscr{P}_{i j}$ также будет Галуа-инвариантно, и по теореме Севери-Брауэра из предложения 3 будет следовать квазитривиальность $X$. Обозначим через $\mathscr{L}_{i}$ линейную систему, определяющую естественную проекцию $S$ на $i$-й сомножитель. Пусть $\mathbb{k}_{i} \supset \mathbb{k}$ - минимальное поле, над которым определена линейная система $\mathscr{L}_{i}$. Выберем (с помощью утверждения 1 ) определенный над $\mathbb{k}_{1}$ дивизор $D_{1}$ вида $H_{1} \times \mathbb{P}^{n} \times \cdots \times \mathbb{P}^{n}$ в соответствующей Галуа-инвариантной линейной системе. Заметим, что орбита $O_{1}$ дивизора $D_{1}$ под действием $G$ состоит из дивизоров $D_{i}$ таких, что при $i \neq j D_{i} \nsim D_{j}$ в группе $\operatorname{Pic}(\bar{X})$. Действительно, в противном случае нашлись бы такой элемент $g \in G$, что $g\left(D_{i}\right) \neq D_{i}$, но $g\left(D_{i}\right) \sim D_{i}$ для некоторого $i$, и такой сопряженный ему элемент $h \in G$, что $h\left(D_{1}\right) \neq D_{1}$, но $h\left(D_{1}\right) \sim D_{1}$. Поскольку в этом случае $h$ тождественно действует на $\mathbb{k}_{1}$, а $D_{1}$ определен над $\mathbb{k}_{1}$, мы приходим к противоречию. Если в орбите $O_{1}$ нет дивизора из линейной системы $\mathscr{L}_{j}$, рассмотрим орбиту $O_{j}$ соответствующего дивизора $D_{j}$ и так далее для всех $j$. Объединив все такие орбиты, мы получим инвариантный центр проекции $L$, что доказывает теорему 2 .

Докажем следующее обобщение теоремы 2.

ПредЛожениЕ 4. Всякая форма $Y$ общего гиперплоского сечения $W=H \cap S \subset$ $\mathbb{P}^{a b+a+b-1}$ многообразия Сегре $S=\mathbb{P}^{a} \times \mathbb{P}^{b} \subset \mathbb{P}^{a b+a+b}$ квазитривиальна.

ДокАЗАТЕЛьство. Заметим, что пара линейных систем, отображающих $W$ на сомножители $\mathbb{P}^{a}$ и $\mathbb{P}^{b}$, является Галуа-инвариантной (группа Галуа может лишь переставлять проекции на разные сомножители в случае $a=b)$. Следовательно, естественное вложение $W \hookrightarrow S$ Галуа-инвариантно, а значит, согласно утверждению 1 , можно считать, что $\bar{Y}=W=H \cap S \subset \mathbb{P}^{a b+a+b-1}$, где $S$ и $H$ определены над $\mathbb{k}$. Рассмотрим общий инвариантный центр $L$ проекции $\pi_{L}: S \rightarrow \mathbb{P}^{a+b}$ и бирациональную проекцию

$$
\pi_{L_{H}}: S \rightarrow Q \subset \mathbb{P}^{a+b+1}
$$

с центром $L_{H}=L \cap H$ на гиперповерхность $Q \subset \mathbb{P}^{a+b+1}$. Над $\overline{\mathbb{k}}$ через каждую точку $p \in L_{H} \cap S$ проходит $(a+b-2)$-мерное семейство подмногообразий вида $\mathbb{P}^{1} \times \mathbb{P}^{1} \subset S$, и при проекции $\pi_{L_{H}}$ общее такое подмногообразие переходит в плоскость на $Q$. Таким образом, $Q$ содержит $(2(a+b)-5)$-мерное семейство плоскостей, а его гиперплоское сечение $Q_{H}=\pi_{L_{H}}(W)$ имеет размерность $a+b-1$ и содержит $(2(a+b-1)-3)$-мерное семейство прямых. Поэтому, согласно [3], гиперповерхность $Q_{H}$ является либо квадрикой, либо рациональным (ввиду рациональности $W$ ) скроллом в $P^{a+b}$. Квазитривиальность квадрики хорошо известна. Пусть $Q_{H}$ является рациональным скроллом. Ввиду общности $L$, из наличия $\mathbb{k}$-точки на $W$ следует наличие гладкой $\mathbb{k}$-точки на $Q_{H}$. В этом случае $Q_{H}$ является расслоением на $(a+b-2)$-мерные проективные пространства над $\mathbb{P}^{1}$, сечение к которому доставляет общее проективное пространство коразмерности $a+b-2$. Таким образом, гиперповерхность $Q_{H}$ квазитривиальна, а значит, многообразие $W$ также квазитривиально.

Автор выражает благодарность С.С. Галкину, С. О. Горчинскому, Ф.Л. Заку, В. А. Исковских, Ю. Г. Прохорову и К. А. Шрамову за полезные обсуждения.

\section{Список литературы}

[1] A. Grothendieck, Inst. Hautes Études Sci. Publ. Math., 1961, № 8, 1-222; 11, 349-511. [2] Ю. И. Манин, М. А. Цфасман, УМH, 41:2 (1986), 43-94. [3] B. Segre, Atti Accad. Naz. Lincei. Rend. Cl. Sci. Fis. Mat. Nat. (8), 5 (1948), 193-197; 275-280.

H. Ф. Зак (N. F. Zak)

Московский государственный университет им. М. В. Ломоносова

E-mail: nzak@mccme.ru
Представлено В. М. Бухштабером Принято редколлегией 06.06.2007 